\title{
THE EFFECT OF ADRENALINE ON THE INTRA-OCULAR PRESSURE AND ITS CLINICAL SIGNIFICANCE
}

\author{
BY
}

W. S. Duke-Elder, P. M. Duke-Elder, and J. C. Colle

(FROM THE DEPARTMENT OF PHYSIOLOGY AND BIOCHEMISTRY, UNIVERSITY COLLEGE, LONDON)

MUCH work has been done upon the action of adrenaline on the pressure of the eye when the drug has been administered as drops in the conjunctival $\mathrm{sac}$, as a sub-conjunctival or retro-bulbar injection, or by the intra-venous route. The importance of the matter from the clinical point of view is considerable, inasmuch as adrenaline has been widely advocated and employed as a measure for lowering the intra-ocular pressure in cases of glaucoma, more particularly of recent years by Hamburger in the synthetic preparation "glaucosan." In the experimental observation of its effects the intra-venous route of administration is the most satisfactory method to employ owing to the rapidity and the constancy of the subsequent reaction, and the present paper deals solely with this aspect of the matter. Our apology for re-introducing the subject is that we believe we have been able to amplify and coordinate the results of former investigators by the employment of more accurate and delicate technique.

The conclusions of those who have investigated the action of adrenaline on the intra-ocular pressure when the drug has been administered by the intra-venous route have been, to a certain extent, indeterminate. In some experiments a rise in the intra-ocular pressure has been obtained, in others a fall, in some a fall and then a rise, and in others no observable change at all. Thus Parsons (1903) obtained a rise in pressure, the intra-ocular pressure runining parallel to the blood pressure; Henderson and Starling (1904) sometimes obtained a rise and sometimes a fall, concluding that it was not permissible to use such experimental evidence as a foundation for any theory as to the action of this drug. Wessely (1908) got somewhat similar results. The explanation offered by all of these was substantially the same; when an increase in the pressure was recorded it was considered due to the rise in general blood pressure making itself evident in the eye ; if a fall was recorded it was due to the local vaso-constriction asserting itself as the predominant feature in the picture and preventing the effect of the raised blood pressure making itself evident in the eye; and to these was added the further factor of a constriction of the plain muscle of the orbit which was particularly stressed by Wessely. These conclusions are all correct so far as they go, but they are susceptible to finer analysis. 


\section{Experimental Methods}

In the experiments detailed in the present paper, two types of procedure were carried out. In the first place intact dogs were employed under ether-chloralose anaesthesia, the intra-ocular pressure being recorded by the manometer described in a previous paper (Vol. XV, p. 575,1931 ), and the blood pressure being registered simultaneously by a mercury manometer connected with the carotid artery on the side opposite to the eye employed. In a second series of experiments records were taken on the eyes of perfused animals. In all the experimental work which has been done hitherto upon the variations of the intra-ocular pressure with drugs, intact animals have been employed, and. it has frequently been difficult to dissociate the effects of general and local conditions. The administration of adrenaline involves a change in the general blood pressure and also

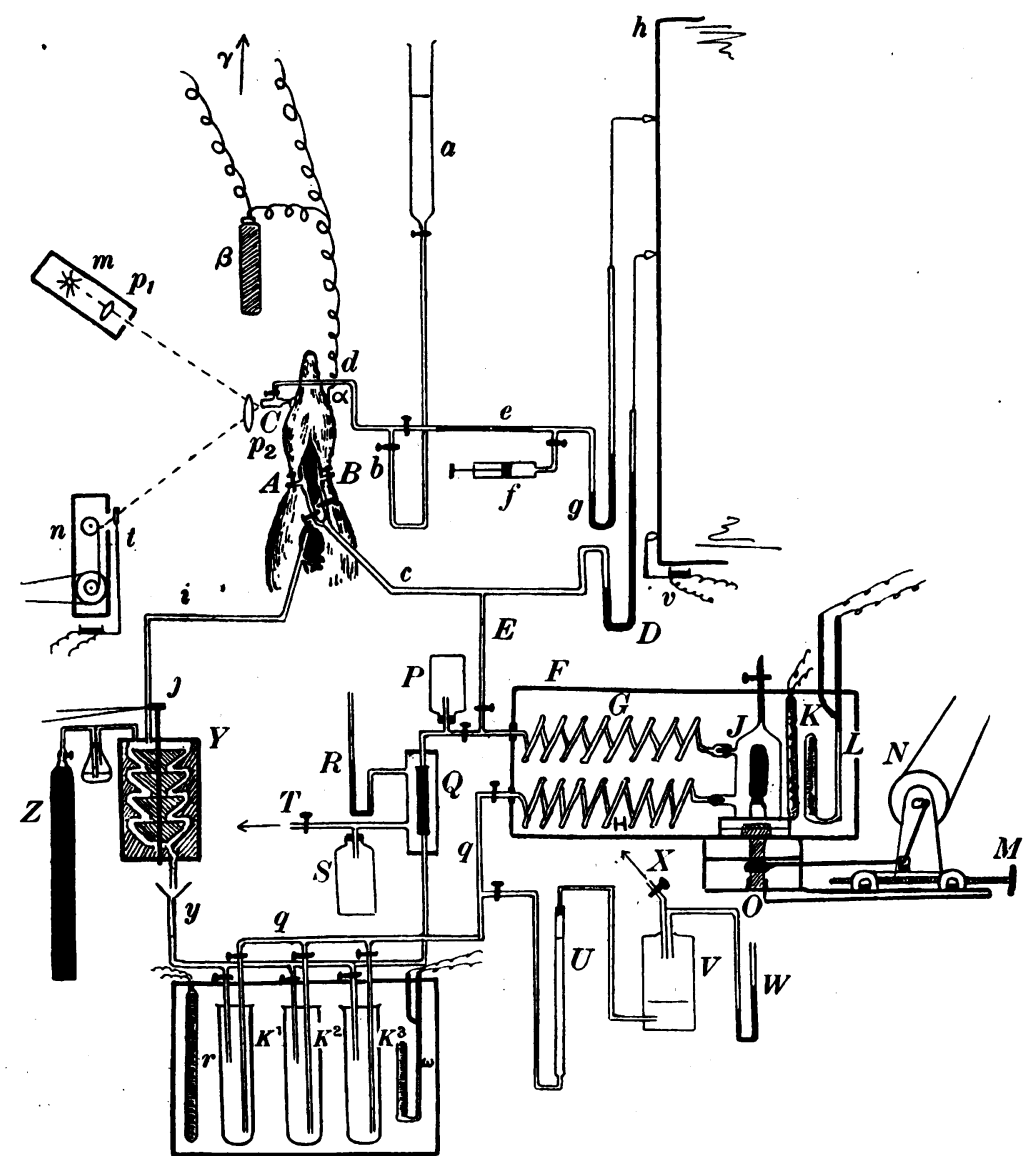

Fig. 1. 
a local alteration in the vessels of the eye itself, and it is essential to eliminate one of these variables if the influence of the other is to accurately assessed. It was, therefore, considered advisable to isolate the eye completely from the influence of the vital centres, and maintain a constant circulation by means of an artificial heart and an artficial lung as in the apparatus seen in Fig. 1.

This apparatus is modelled essentially on that already described by us (DukeElder, 1929), but was improved considerably from experience : a minute description will therefore be unnecessary here. The same type of heart pump was employed (G, H, J, K, L, M, N, O) which pumped blood from one of the reservoirs $\left(K_{1}, K_{2}\right.$. $\mathrm{K}_{3}$ ), while the temperature was kept constant by electrically controlled thermostats. Between the pump and the dog a mechanism was inserted to maintain the blood pressure at a constant level despite any changes in the peripheral circulation of the dog's head. It consisted essentially of a side tube delivering into a length of soft rubber tubing $(Q)$. This was enclosed in a cylindrical glass jacket in which air was maintained at the pressure level it was determined to maintain (100 $\mathrm{mm}$. $\mathbf{H g}$ ) by pumping air through the side tube $(T)$ and the pressure bottle $(S)$, the rise of the pressure being registered on the mercury manometer (R). The heart pump was so adjusted that the pressure in the circulation was slightly above the required level so that blood, after passing through the inverted bottle $(P)$ wherein the pulse beat of the pump was damped down to simulate the normal peripheral resistance of the circulation, arrived at $(Q)$. Since the air in the jacket compressed the rubber tube (Q) up to the limit of $100 \mathrm{~mm}$. Hg any excess of blood above this pressure forced its way through the tube and was short-circuited back to the reservoirs, so that the volume of blood reaching the head was maintained at a rigorously constant pressure level. On leaving the head through the tube (i) on its way to the reservoir, the blood was areated by passing through an artificial lung (Y) of the type devised and described by Bayliss, Fee, and Ogden (1928). Here it fell in cascades over a series of discs rotating in opposite directions, and in the meantime was exposed to a stream of a physiological mixture of oxygen and carbon dioxide fed from the cylinder $(Z)$. A second improvement was represented by a constant infusion apparatus $(U, V, W)$ of the type described by Burn and Dale (1926), by which a minute and constant supply of adrenaline was added to the circulating blood. This is necessary when studying vascular reactions in order to maintain the tone of the capillaries and replace the influence of the hormones of the normal animal. The amount of adrenaline constantly added was 1 c.c. of a 1 in 50,000 solution in physiological saline every five minutes. In place of decapitating the animal, as was done in former experiments, it was found that the condition of the head was improved by retaining it intact. The artificial circulation was introduced through the carotids without allowing any hiatus in the blood flow by the method described in the previous paper (Duke-Elder, 1929), and after the whole of the collateral circulation had been excluded by ligation of the appropriate vessels in the thorax, the venous blood was collected through the superior vena cava. As before the vitality of the head was maintained unimpaired for periods of several hours; the corneal reflex was present, the pulse beat was retained on the curve of the intra-ocular pressure, and the intra-ocular circulation was normal on ophthalmoscopic examination.

One of the factors which constantly tends to obscure vascular changes in the eye when these are deduced from variations in the intra-ocular pressure is the action of the extra-ocular musculature. This consists of two components : the plain muscle of Müller which protrudes the eye causing an exophthalmos, and the striped muscle (recti and obliques) which, when acting as a whole, tend to pull the eye inwards causing an enophthalmos. A contraction of either of these sets of muscles raises the pressure of the eye, and a relaxation of their tone induces a fall. Since the head of the experimental 
animal was securely clamped in the same system as the optical manometer, any movement of the eye relative to the head was registered on the tracing of the intra-ocular pressure by a movement of the base line, a movement in one direction denoting an exophthalmos, in the other an enophthalmos. Movements of the voluntary muscles were eliminated by curare.

Two other collateral means of investigation were employed to aid in the interpretation of the results. In order to correlate variations in the intra-ocular pressure more exactly with events in the intraocular circulation, the rate of blood-flow in the eye (that is, essentially the state of dilatation of the arterioles) was measured by recording variations in the temperature. An insulated constantan wire was soldered into a hypodermic needle, the end of which was re-sharpened; this was thrust through the cornea into the anterior chamber of the eye which was not employed for manometric registration $(a)$. This thermo-junction was balanced against a similar junction in a thermos flask $(\beta)$ and connected with a microgalvanometer $(\gamma)$, the deflections of which were registered optically. When lying in the anterior chamber the needle excites no disturbance soon after its insertion, and changes in the flow of blood can be directly correlated with changes in the temperature. A typical reaction, for example, is a rapid fall of $2^{\circ}$ to $4^{\circ} \mathrm{C}$. which follows the occlusion of the carotid artery on the same side as the eye experimented upon, or a rise of the same order which follows occlusion of the abdominal aorta which diverts an excess of blood towards the head.

Confirmation of the events occurring in the local vascular system was obtained by the direct observation of the vessels of the iris of albino rabbits. It is not possible to observe these changes accurately in any but an unpigmented eye, and consequently dogs (which were used in the artifichal perfusion) and cats (which were sometimes used in the experiments on intact animals) were unsuitable for this purpose. These observations had therefore to be undertaken on albino rabbits which were used anaesthetized by intra-peritoneal urethane. The head of the animal was immobilized in a convenient position, and when the drug in question was injected into the circulation, the vessels of the iris were observed through a $Z$ eiss binocular microscope in the focal beam of a Gullstrand slit-lamp. At the same time an estimate of the degree of permeability of the vessels was based on the appearance of trypan blue in the aqueous humour after its intra-venous injection. In the normal animal the capillaries of the eye are impermeable to this dye; it is only detected in the anterior chamber when the permeability of the minute vessels has been increased by a very definite degree, when the presence of a minute amount in the anterior chamber is readily seen in the optical conditions employed. 
The appearance and distribution of the minute vessels in the eye of the albino rabbit have recently been studied in considerable detail by Larsen (1930), and we were able to confirm his findings. The major circle of the iris is clearly seen as a large artery running a sinuous course round the periphery of the iris near the angle of the anterior chamber; it is fed directly by the long posterior ciliary arteries. It sends off a series of arterial twigs which run towards the pupillary margin, where a number of small arterial branches of arteriolar dimensions are seen under high magnification lying superficially in the stroma of the iris in the neighbourhood of the sphincter muscle. Apart from this, in normal conditions the surface of the iris is practically avascular; but when the capillaries are dilated the entire surface becomes dotted in varying degree with minute capillary loops. It is thus possible to observe directly three types of vessels and study their reaction under varying conditions: an artery (the greater circle), arterioles near the pupillary margin, and capillary loops upon the surface of the iris.

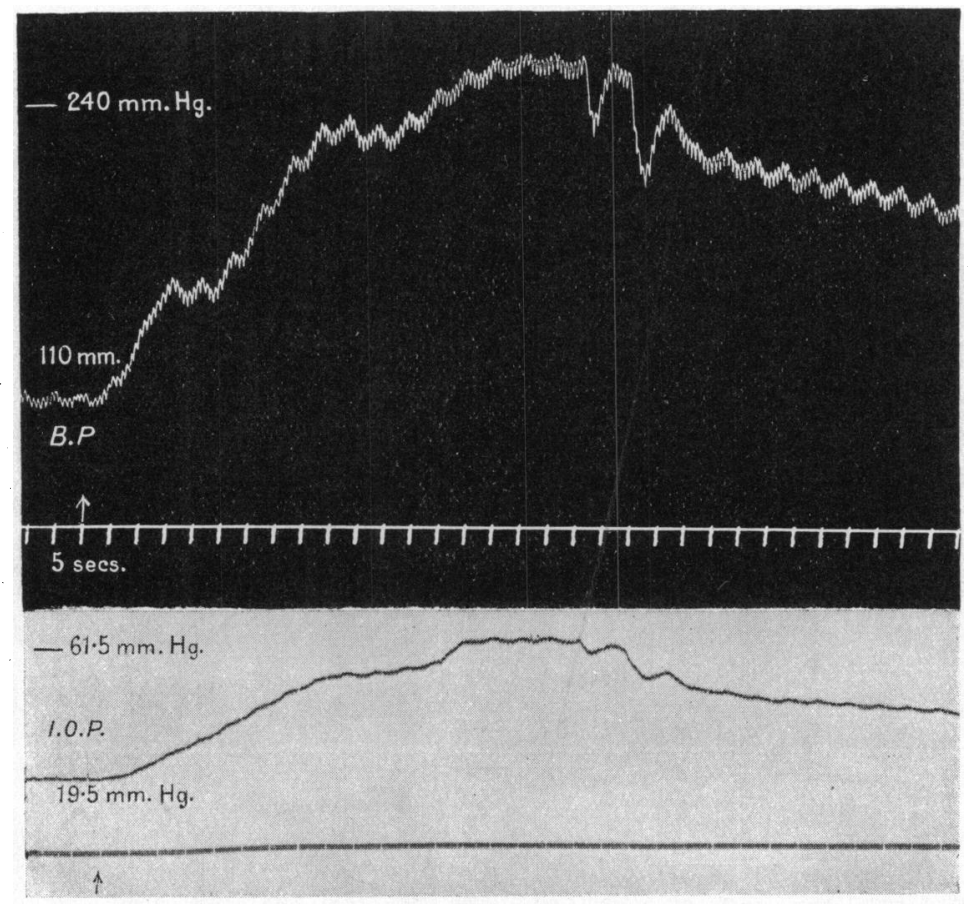

FIG. 2.

The effect of the intra-venous injection of 01 c.c. of 1 in 10,000 solution adrenaline in the intact dog. Upper curve, the blood-pressure; lower curve, the intra-ocular pressure. 


\section{Experimental Results}

In experiments on the intact animal three types of results are obtained : (1) With small doses $(0 \cdot 1$ c.c. of 1 in 10,000 solution and anything under this which produces an effect) the blood pressure is raised and the intra-ocular pressure tends to be raised passively with it, the amplitude and characteristics of the pulse being conveyed to the curve of the pressure of the eye (Fig. 2). This is essentially a passive effect, and would seem to show that any pressor action in the eye-is less readily evident than upon the heart and those parts of the body, such as the skin, in which arteriolar constriction contributes to the general rise of blood pressure after the administration of adrenaline. (2) With large doses (of the order of 1 in 1,000 solution) the rise in general blood pressure is accompanied by a definite fall in the intra-ocular pressure during which the amplitude of the pulse is very considerably throttled down, suggesting that a considerable amount of vaso-constriction is occurring in the eye. Frequently these two effects are to some extent combined, and while the blood pressure rises the intra-ocular

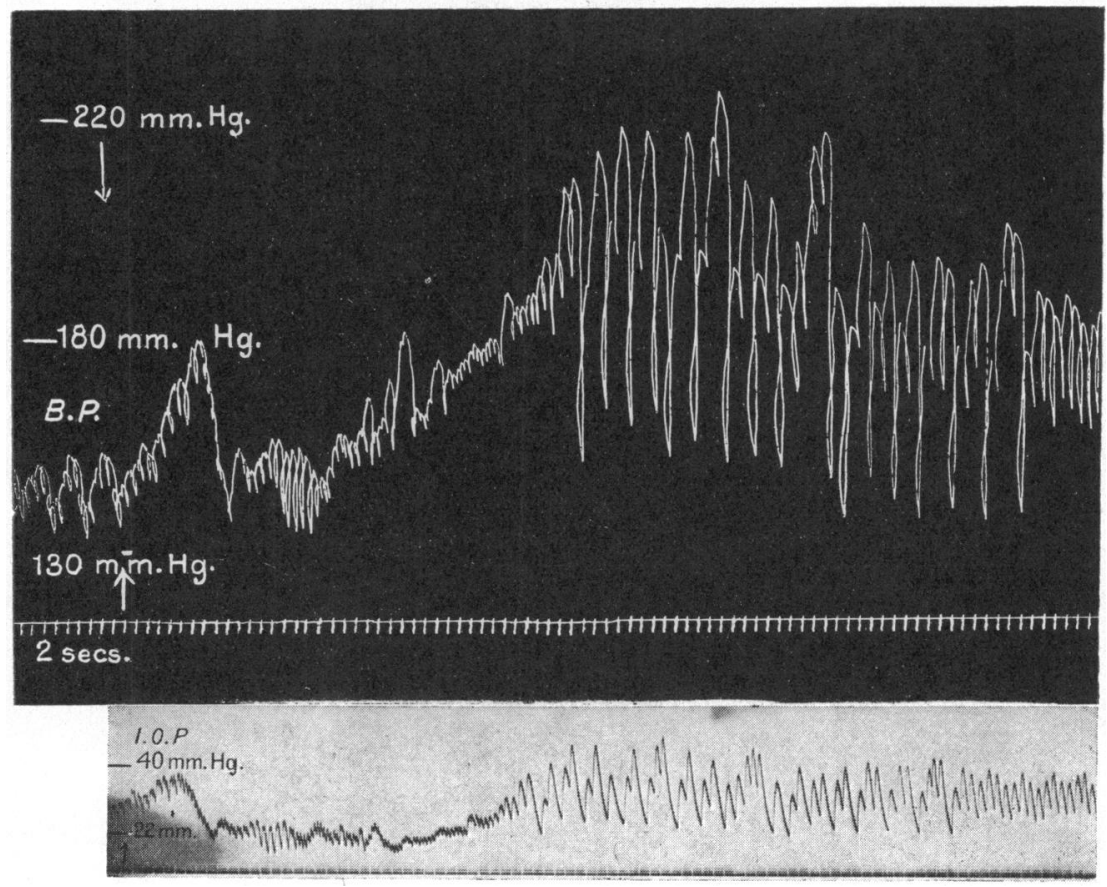

Fig. 3.

The effect of the intra-venous injection of $0 \cdot 1$ c.c. of 1 in 1,000 solution of adrenaline in the intact dog. 
pressure falls initially, but when the general pressor reaction has reached its height, the barrier of arteriolar constriction in the eye is broken down and the intra-ocular pressure consequently rises, the ocular pulse showing a large excursion : again, the barrier offered by arteriolar constriction in the eye is relatively feeble (Fig. 3). These vascular events were confirmed by observations on the temperature in the anterior chamber, a rise of pressure being accompanied by a raising of the temperature by $2^{\circ}$ to $3^{\circ} \mathrm{C}$., and a fall of pressure being indicated by a decrease in temperature suggesting arteriolar constriction.

(3) A type of reaction where a persistent rise of the intra-ocular pressure occurs quite independently of any variation in the general blood pressure and associated with no characteristic alteration of the pulse beat is occasionally observed. This type of reaction is well illustrated in a paper by Wessely (1908), and is presumably due to the contraction of the plain muscle of Müller. Such a reaction is accompanied by no constant temperature variation.

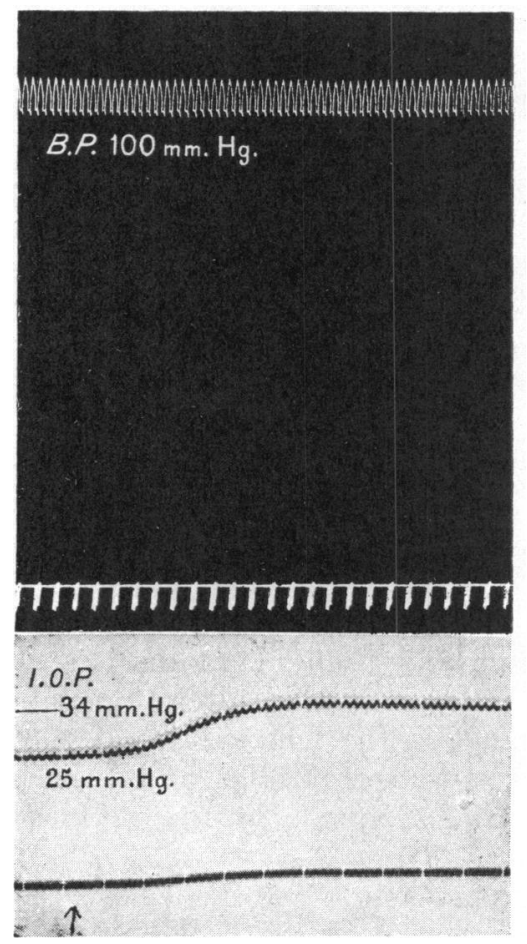

FIG. 4.

The effect of 0.2 c.c. of 1 in 10,000 solution of adrenaline in the blood of the perfused dog. The movement upwards of the base line in the tracing of the intra-ocular pressure denotes an exophthalmos. 
These various effects can be analysed with much more accuracy by a study of the artificially perfused eye. With this preparation the almost invariable result of a small dose of adrenaline under 0.1 c.c. of a 1 in 10,000 solution is to give a rise in intra-ocular pressure. The rise in pressure may be of two types which may be due to different causes.

(1) An abrupt rise in pressure occurs which is associated with the movement of the base line in the direction of exophthalmos which is retained after curare (Fig. 4). The rise of pressure rapidly

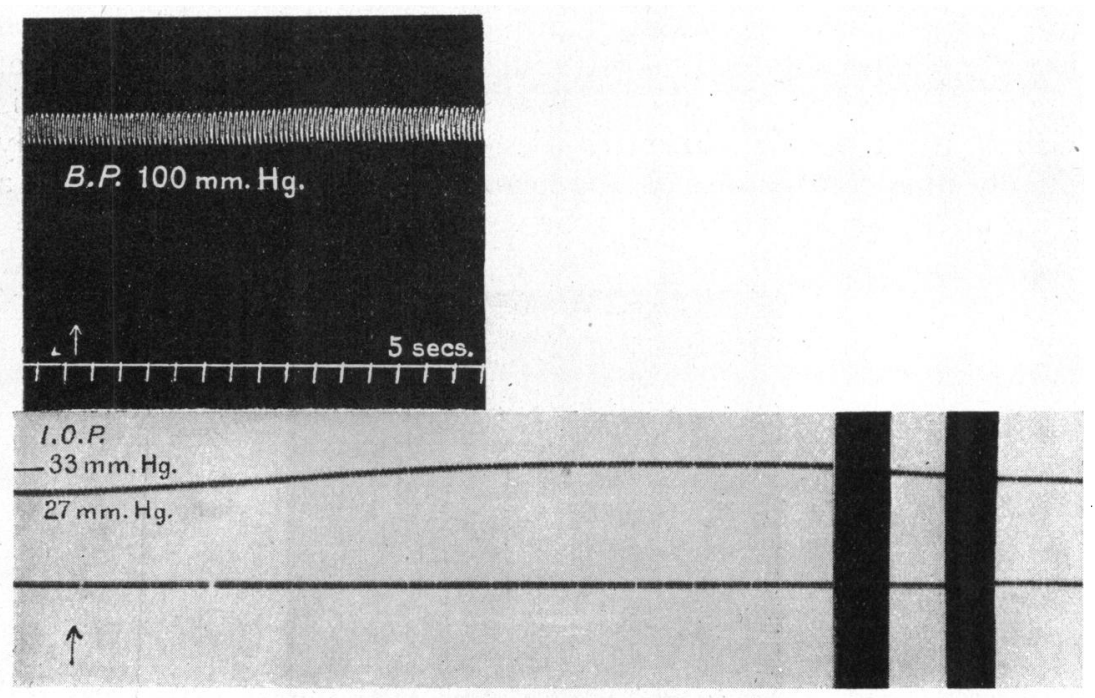

Fig. 5.

The effect of 0.1 c.c. of 1 in 10,000 solution of adrenaline in the blood of the perfused dog. The breaks in the tracing of the intra-ocula: pressure denote 1 minute intervals. The blood pressure was maintained constant throughout.

attains a maximum and then maintains a constant level, or falls slowly being subject to no rapid alterations, and it is not accompanied by any change in the temperature of the eye. It is due to contraction of the muscle of Müller, and it is interesting that this effect is much more frequent in the perfused dog than in the intact dog.

(2) A second type of rise occurs which is of a completely different - nature wherein the rise of pressure slowly and gradually attains a maximum and then falls (Fig. 5). It is accompanied by no change in the base line and no movement of the eye, and by a slight rise in the temperature of the aqueous humour $\left(0^{\circ} 25^{\circ}\right.$ to $0.5^{\circ} \mathrm{C}$.). The type of curve has all the characteristics which we have become 
accustomed to associate with a vascular change, and since the rise of temperature is comparatively small, it appears unlikely that it is dependent on any great alteration in the arterioles. On the other hand, examination of the intact eye of the rabbit after the administration of adrenaline in such doses shows that the minute vessels on

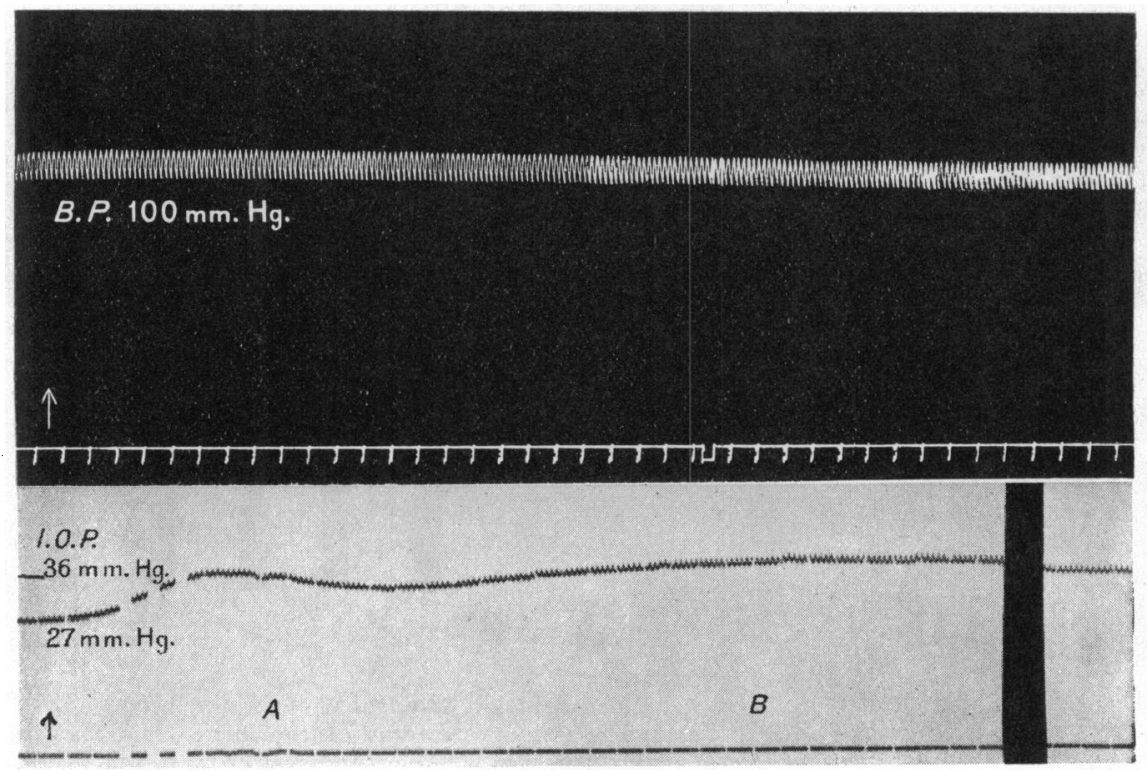

FiG. 6.

The effect of 0.15 c.c. of 1 in 10,000 solution of adrenaline in the blood of the perfused dog. The first rise in the intra-ocular pressure (A) is due to a contraction of Müller's muscle; the second (B) to capillary dilatation.

the anterior surface of the iris were dilated, and that their permeability is increased as is shown by the passage of trypan blue into the anterior chamber (see also Larsen, 1930). We consider therefore that this type of response is due to capillary dilatation; so far as we are aware it has hitherto escaped observation.

It occasionally happens that these two types of response are combined, the first rapid rise being due to the action on the external musculature, and the second, slower rise, being due to the dilatation of the capillaries (Fig. 6).

With larger doses of adrenaline (of the order of 0.1 c.c. of 1 in 1,000) a movement of exophthalmos with an associated rise in the intra-ocular pressure almost invariably occurs which, however, is more than counterbalanced by a fall in pressure (Fig. 7). The appearance in the iris of the rabbit of vaso-constriction is confirmed 


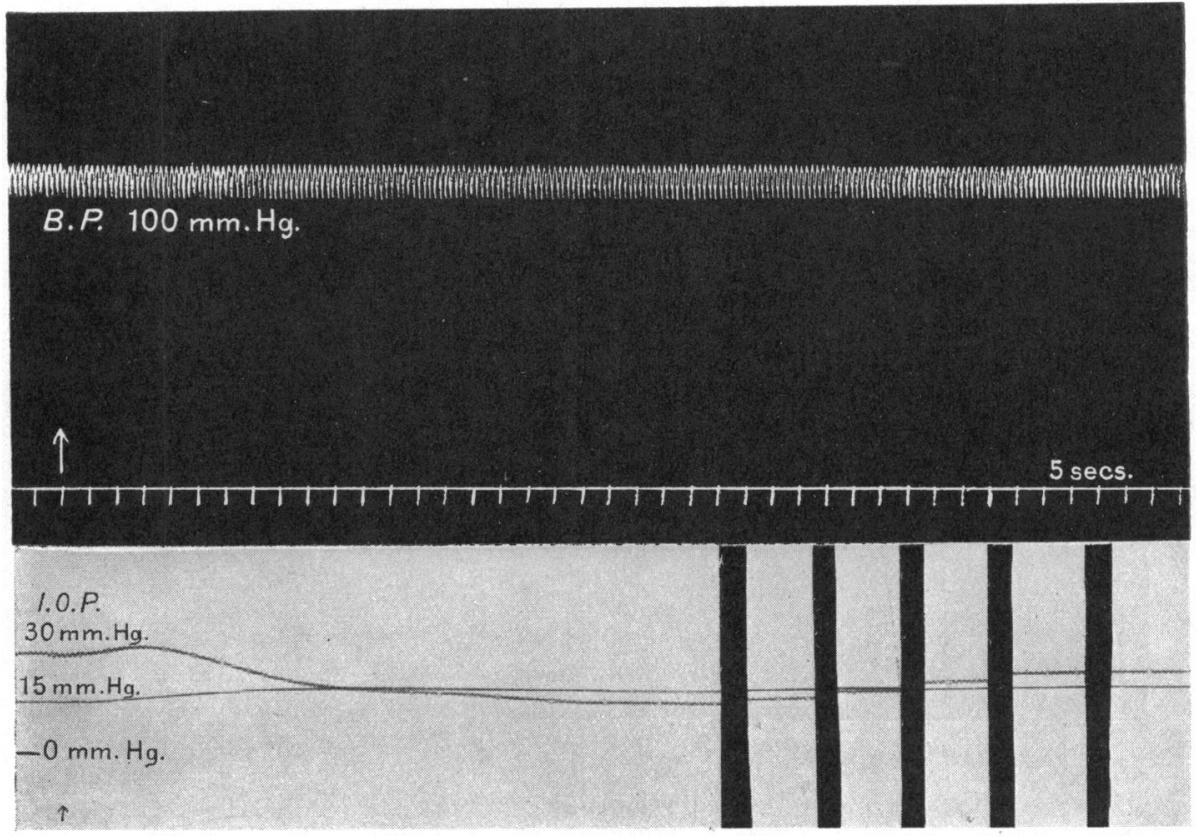

Fig. 7.

The effect of 0.1 c.c. of 1 in 1,000 solution of adrenaline in the blood of the perfused dog. The spacings in the tracing of the intra-ocular pressure represent $2 \mathrm{~min}$. intervals. The movement upwards of the base line denotes an exophthalmos. Note the initial rise in the intraocular pressure followed by a fall.

by temperature records which show a definite fall $\left(2^{\circ}\right.$ to $3^{\circ} \mathrm{C}$.). This is obviously due to arteriolar constriction, and apparently this has become so pronounced that it can bring about a fall in pressure which is sufficient to counterbalance the rise due to muscular contraction.

\section{Conclusions}

It appears, therefore, as judged from pressure records in the intact animal and the artificially perfused eye, from the change of blood-flow as recorded by temperature changes, and from the direct observation of the intra-ocular vessels, that the action of adrenaline upon the eye itself uncomplicated by the effects of the general circulation is four-fold ; first, in small doses, it dilates the capillaries and raises the intra-ocular pressure; second, in large doses it constricts the arterioles and capillaries and lowers the pressure; third, in any dose it constricts the plain muscle of the orbit and 
raises the pressure; and fourth, it dilates the pupil, an action which appears to be without any significant influence on the intra-ocular pressure, since it may be associated with either a rise or a fall. In the intact animal, where the situation is complicated by general vascular changes, the raised blood pressure tends to break through any arteriolar resistance which may be present and causes a rise in the intra-ocular pressure unless (with individual variations) the dosage is very high, when by local constriction of the arterioles may be sufficient to counterbalance this.

In man the action of Müller's muscle in raising the intra-ocular pressure will be very much less than in the dog, owing to its very small development in the former; it is probable indeed that it may be neglected altogether. Apart from this, the present investigation accounts for the variability of the action of adrenaline upon the pressure of the eye. Depending upon the dose in which it becomes effective in the eye it will either dilate the minute vessels raising the pressure, or constrict them tending to lower the pressure, and the possibility of the constriction becoming effective will depend upon the extent to which the drug is absorbed into the general circulation. The fact that the vessels of the eye react less markedly than those of large areas elsewhere in the body (particularly in the skin, and upon the heart itself) makes ocular changes subservient to general changes. A further complication arises which can only be referred to briefly at present. After the minute vessels have been exposed to the action of histamine-like substances they undergo a refractory phase wherein adrenaline is without constrictive effect. How far this may account not only for the frequent failure of adrenaline to produce a hypotensive effect in cases of acute glaucoma, but also for the fact that it may raise the pressure still further, will be made the subject of a future communication.

\section{REFERENCES}

Bayliss, L. E., Fee, A. R., and Ogden, E.-Jl. of Physiol., Vol. LXVI, p. 443, 1928.

Brown, J. H., and Dale, H. H.-Jl. of Physiol., Vol. LXI, p. 185, 1926.

Duke-Elder, W. S. and P. M.-Brit. Jl. of Ophthal., Vol. XIII, p. 385, 1929 ; Vol. XV, p. 575, 1931.

Henderson, E. E., and Starling, E. H.-Jl. of Physiol., Vol. XXXI, p. 305, 1904.

Larsén.--Ueber den Augendruck u. die vorderen intraokularen Gefässe. Stockholm, 1930.

Parsons, J. H.-The Ocular Circulation. London, 1903.

Wessely, K.-Arch. f. Augenheilk., Bd. LX, SS. 197, 1908. 